\title{
Identidad, tiempo y espacio en El tornavoz de Jesús Gardea
}

\author{
Angélica TORNERO SALINAS \\ Universidad Nacional Autónoma de México \\ Universidad Autónoma del Estado de Morelos
}

\begin{abstract}
En este artículo se explora la relación entre tiempo, espacio e identidad en $E l$ tornavoz de Jesús Gardea. El objetivo es observar cómo se entrecruzan el espacio y el tiempo y cómo esta relación se resuelve en la configuración de la identidad de los personajes. La reflexión se realiza en dos dimensiones, a partir del análisis de las estructuras del texto y de la hermenéutica.
\end{abstract}

PALABRAS CLAVE: espacio, tiempo, identidad, hermenéutica.

In this article the relationship between time, space and identity in El tornavoz by Jesús Gardea is explored. The main aim is to observe how space interacts with time, and the effect that this relationship produces in the configuration of the identity of characters. This revision is carried out from two perspectives: structural analysis and hermeneutics.

KEY WORDS: space, time, hermeneutics, identity.

En los siglos XVIII y XIX, la física teórica y la experimental se encargarán de pensar el espacio mucho más de lo que lo hará la filosofía, la cual se abocará principalmente al pensamiento sobre el tiempo. Ya Foucault señaló la descalificación del espacio a lo largo de décadas: "el espacio es lo que estaba muerto, fijado, lo no dialéctico, inmóvil. Por el contrario, el tiempo era rico, fecundo, vivo, dialéctico" (Foucault, 1992: 126). Hacia la segunda mitad del XX, el espacio recobrará actualidad en el marco de la teoría literaria, asociado a la cuestión del tiempo (Garrido, 1996: 208), no sólo por la primicia en el movimiento romántico de la imagen y sus implicaciones espaciales, sino también por las repercusiones que esta perspectiva tuvo en la narrativa.

Los cuentos y novelas de vanguardia y algunas expresiones llamadas posmodernas encontraron en el espacio su anclaje estético. Uno de los objetivos de estas obras fue, precisamente, cuestionar el tiempo y, con ello, a la narración misma, como estrategia fundamental para la configuración de relatos. Esta importante perspectiva, que introduce una paradoja en el pensamiento sobre las narraciones, ha abierto nuevas líneas de reflexión en la teoría de la literatura. La prioridad del espacio sobre el tiempo en estas aproximaciones narrativas implica un cambio en la concepción misma de la relación 
entre estas dimensiones, con implicaciones ontológicas. Los enfoques postestructuralistas y posmodernos han insistido en el privilegio del espacio en la literatura como presentación y no como representación, como presencialización; dicho de otro modo, como cristalización de la acción narrativa. Algunas novelas cercanas al nouveau roman, definido por esta estética, se inclinan por esta propuesta, con lo que no sólo se desdibuja la temporalidad, sino también la identidad de los personajes (Ricardou, 1971). Como es sabido, esto implicó el cuestionamiento del yo, del sujeto y de la identidad, y con ello, del autor, de la voz narrativa e incluso del lector.

En este artículo se explora la relación entre tiempo, espacio e identidad en $E l$ tornavoz del escritor chihuahuense Jesús Gardea. La intención es observar cómo se entrecruzan el espacio y el tiempo y cómo esta relación se resuelve en la configuración de la identidad del que habla. La reflexión se realiza en dos dimensiones, a partir del análisis de las estructuras del texto y de la hermenéutica.

El tornavoz (1984) es la tercera novela escrita por Jesús Gardea, tras haber incursionado en el cuento y en la poesía. Este escritor mexicano nació en Delicias, Chihuahua, en 1939, y murió en el Distrito Federal, en marzo de 2000. Su obra abreva de obras de autores fundamentales como Juan Rulfo y Gabriel García Márquez, sólo por señalar a dos escritores relevantes en el ámbito hispanoamericano.

\section{Placeres: ciudad del displacer, ciudad del olvido}

El tornavoz simula narrar la saga de tres generaciones de hombres que llevan el apellido Paniagua. Se trata de una parodia, porque no se relata a la manera de una leyenda o fábula realista, sino de una imitación de este discurso. ${ }^{1}$ Si se tomara en cuenta la edad de los diferentes personajes, con el supuesto de que se narra la saga de tres generaciones, se podría concluir que en esta novela se abarca alrededor de cien años. Hay, no obstante, elementos que permiten pensar que no es así. La intención del texto ${ }^{2}$ es expresar la soledad de un hombre, que en vida padece la incomprensión de los demás y que, ya muerto, busca resonar, hacerse oír entre los vivos o, en otro sentido, ser recordado. Se narra cómo Cándido Paniagua se introduce en el mundo de los vivos y cómo los vivos reciben a este muerto; dicho de otro modo, qué pasa cuando estos dos mundos se intersectan.

En la novela es posible distinguir dos dimensiones espaciotemporales, que podríamos denominar mundo de los muertos y mundo de los vivos. En el primero, Cándido,

\footnotetext{
${ }^{1}$ No se trata de la parodia entendida en sentido burlesco o peyorativo. Linda Hutcheon ha señalado este carácter no peyorativo de la parodia, sobre todo en la ficción posmoderna, y su intención crítica en relación con el texto o género parodiado (Hutcheon, 1992: 182).

${ }^{2}$ No hablo de la intención del autor, por lo que no hay que confundir con la falacia intencional. Siguiendo a Ricoeur, me refiero a la intención del texto: "Lo que se ha de comprender de un relato no es en primer lugar al que habla detrás del texto, sino aquello de lo que se habla, la cosa del texto, a saber, el tipo de mundo que la obra despliega delante del texto" (Ricoeur, 1986: 155).
} 
ya muerto, va de un sitio a otro y se comunica con muertos y vivos. En el segundo, los vivos, Isidro y Jeremías, sobrino y sobrino nieto de Cándido, respectivamente, viven una temporalidad en apariencia vulgar, pero la incidencia de Cándido en el mundo de estos personajes provoca confusión en relación con su propia situación existencial. Hay un presente efectivo del relato que abarca del nacimiento de Jeremías a su partida de Placeres a los diez años y a su regreso veintisiete años después, y un conjunto de microrrelatos de los personajes involucrados, no yuxtapuestos sino relacionados con la historia principal.

Cándido Paniagua es la voz primordial, cuya situación se imbrica en este presente del relato, estando vivo y muerto. Podría decirse que, en el marco de esta superposición de dimensiones espaciotemporales, se narra la historia de Cándido cuando es joven y adulto y cuando se convierte en una "voz" que desea ser escuchada. Se cuenta también la infancia de su sobrino, Isidro, y su etapa de adulto y el nacimiento de su hijo, Jeremías, y la etapa de adulto de este último. Cándido Paniagua cruza las generaciones que vienen detrás de él y "sigue vivo", como voz, dentro de Jeremías.

Esta configuración temporal se resuelve en el discurso en tres secciones, señaladas con números romanos. En la primera, la presencia de Cándido es evidente y constante, y aparece en relación con Isidro niño. Aquí, la historia se imbrica con la de Isidro adulto. El narrador va de un tiempo a otro. Pero no sólo esto, también se imbrica con la de Jeremías, porque el niño ha nacido ya. Es preciso señalar que las tres secciones en las que se divide la novela están, a su vez, divididas en muchas más, por espacios en blanco dejados en el texto. En el primer segmento de esta primera parte aparecen los tres personajes: Isidro está con sus amigos espiritistas, específicamente con Omar Vitelo, evocando a Cándido ya muerto, cuando nace Jeremías. En la segunda sección, Cándido es una voz que busca resonar en los demás; todo gira alrededor de él, aun cuando no está presente. Cándido es el personaje que permite estructurar el conjunto de microhistorias que constituyen esta novela; las pequeñas historias que derivan, motivadas por este personaje. En la tercera sección, Cándido intenta volver al mundo a través de Jeremías.

La construcción temporal de esta novela es circular. Cándido muerto busca resonar en las generaciones posteriores a él, sin éxito. Esta circularidad genera el efecto de inconclusividad, lo cual, a su vez, indica que este movimiento se perpetúa. Todos los seres humanos cumplen su ciclo vital, que concluye con la muerte y todo se repite, aparentemente, sin sentido. Con esto se confirma la idea de que la construcción de la saga de las generaciones no es sino una parodia.

Nos encontramos, así, con la intersección de dos temporalidades: una que simula linealidad y otra que detiene el paso del tiempo. La primera se expresa precisamente en este simulacro de saga; la segunda, en el tiempo de las acciones que ocurren en una dimensión humana que no requiere el movimiento o desplazamiento físico. Es decir, los personajes expresan sus modos de pensar, acciones del pensamiento y sus modos de sentir, acciones afectivas, y las acciones que implican movimientos físicos se reducen. Así, esta forma de construcción de acciones se aleja del tiempo y espacio entendidos como sucesos, el primero, y como dimensiones geométricas, el segundo. 
Esta complejidad estructural de la temporalidad impide comprender la identidad de personajes en términos de sucesos; son pocas las acciones que se narran. Tampoco puede decirse que los aspectos psicológicos destacan sobre la trama. La complejidad de esta novela radica en la imbricación de dos espaciotemporalidades: la de los vivos y la de los muertos, la cual, además, ocurre a partir de una estrategia de deconstrucción que provoca polisemia en los polos de la dicotomía. La distinción vivo/muerto se desestructura y los valores de uno y otro pierden su lugar definido dentro de la simbólica del catolicismo, desestabilizando el sentido. ¿Son los vivos los muertos? Los vivos y los muertos comparten características y unos son comprendidos a partir de los otros. Esto significa que no son absolutos y que no son excluyentes.

Una construcción como ésta pone en riesgo cualquier teoría sobre la coherencia de la trama, basada en el encadenamiento causal de sucesos. La identidad de los personajes, es decir, que el lector comprenda quién es ése que habla, se relaciona con la configuración del espaciotiempo - que es variable - en el que los personajes van tramando su consistencia, no unívoca, sino equívoca y hasta ambigua.

El espacio juega un papel fundamental para restar abstracción a la identidad de los personajes, porque el lector no se topa con conceptos universales, propiciados por el encadenamiento causal, que prescinde de las especificidades de los caracteres, sino con situaciones existenciales, con acciones que ocurren en un espacio determinado. Esto tampoco significa que prevalezca una propuesta en la que los caracteres destaquen por sus rasgos psicológicos; antes, sobresalen por su situación, por su propia existencia en el mundo, como seres incompletos, expuestos a los avatares de circunstancias que no están bajo su control.

El espacio y el tiempo se expresan también de manera circular: el pueblo donde tiene lugar la mayor parte de las acciones inicia y termina de la misma manera, como un llano en el que reina la soledad: "Mira, en Placeres, un día volverá a reinar el llano, la soledad, como en un principio" (Gardea, 1984: 49). ${ }^{3}$ Esta estructura circular se vincula, en general, con dos referencias fundamentales: la tierra, que es el páramo desértico, y el cielo, al que pertenece Cándido muerto, como se verá en el siguiente inciso. Se prescinde de coordenadas y destacan sólo estas dos posibilidades, cielo/tierra, pero estos sitios no son definidos de manera absoluta, sino que se deconstruyen; es decir, se cuestiona la oposición irrestricta.

El espacio principal de esta novela es el pueblo denominado Placeres. Este pueblo carece de coordenadas geográficas; no se utilizan referencias concretas para determinar su ubicación. La escasez de descripciones lo convierte en un sitio indeterminado (Villamil, 1996). El lugar podría estar ubicado en cualquier punto de la geografía, no sólo mexicana, sino de aquellos páramos desérticos: es un sitio en el que prevalece un clima extremo y hay casas, calles, una bodega, una plaza. En este sitio "Soplaban rachas de aire helado que levantaban la tierra de la calle y chillaban como demonios

\footnotetext{
${ }^{3}$ Todas las citas han sido tomadas de Jesús Gardea, El tornavoz. México, Ediciones Sin Nombre, 2004.
} 
en las esquinas". "[...] Los vidrios de la ventana estaban fríos como un hielo y arrojaban su relente al rostro de Olivia" (54). Y más adelante se lee: "Era fines de marzo. Pero el sol tenía ya el tamaño de uno de verano, y quemaba mucho" (55).

Los elementos constitutivos del espacio son comunes a prácticamente todos los poblados en circunstancias climáticas semejantes. Lo que singulariza a este lugar son las acciones de los personajes relacionadas, como se ha dicho ya, con su propia identidad. Es decir, los constitutivos del espacio en sí mismos no son relevantes; estos elementos carecen de significado desde el punto de vista puramente material. Lo importante en términos de la refiguración del lector es la manera en que los personajes viven este lugar. En otras palabras, el lector interpreta el espacio en relación con la subjetividad de los personajes. El espacio interior y el exterior están estrechamente relacionados, de ahí que se relativice el espacio geométrico (Ainsa, 2005: 35).

En la configuración de este espacio destacan diversas estrategias, relacionadas con la expresión de condiciones climáticas y su vinculación con las sensaciones. Por un lado, la novela se aleja de las consideraciones realistas de configuración del espacio, como describir a partir de esquemas dimensionales. Los referentes lógicos para la distribución del espacio son escasos. El lector no ubica fácilmente la relación entre el lugar donde se encuentra la casa del Isidro y la bodega donde se llevaban a cabo las sesiones espiritistas, la placita o el sitio donde está la casa de Omar Vitelo. Destaca la descripción del espaciotiempo a partir de la mención de las estaciones. La novela inicia así: "Jeremías Paniagua vino al mundo en la madrugada de un verano...." (9); una noche que "no tenía luna ni perros" (9) y fue concebido en "una noche de invierno. Dulce y casi interminable; con viento helado en las ramas de un árbol..." (9). Y un poco más adelante se lee: "En las bocacalles, el calor era menos" (10). En estos primeros párrafos se expone el esquema que configurará la mayor parte de la novela en relación con la creación de la atmósfera: verano/invierno; calor/frío. Esta ambientación del espacio, configurada metafóricamente, crea una situación de permanente desasosiego. Como han observado varios críticos (Villamil, 1996), (Drew, 1985), el nombre del lugar, Placeres, es una denominación irónica, ya que dista de ser placentero o gozoso; es una especie de antiparaíso.

Por otro lado, el clima configura el sentido del espacio y también de la subjetividad de los personajes. La atmósfera de Placeres es asfixiante generalmente y el sol intenso y el calor crean un ambiente denso, en el que resulta difícil moverse: "Estaba denso el sol y la mirada de Vitelo se quebró en el bloque de luz maciza que llenaba y aplastaba la calle. [...]. Iban como arropados por el polvo y el aire caliente, que les impedía la franca respiración y les velaba las cosas" (23). Esta metáfora revela una manera difícil de vivir e incluso de comprender lo que se vive. Más adelante se lee: "En Placeres, están muertos todos los ruidos, ahogados por la calma y el calor". [...] "La soledad de la calle es enorme, como si no hubiera ya mundo. Polvo y sol nada más" (72). El silencio que priva en el lugar implica la falta de actividades de los personajes y por lo tanto la inmovilidad, la parálisis en la que se encuentran. La repetición de la relación entre el sol y su efecto irrecusable, el calor, reitera esta sensación aplastante en el lector, que 
se convierte en imposibilidad de vivir: "Por la ventanilla del autobús [Marta] no ve otra cosa que soledades castigadas hasta la muerte por el sol" (75).

El lector se enfrenta con una estrategia de construcción del espacio que interpela su propio cuerpo. Es de sobra sabido que no se puede hablar de percepción en la literatura como se hace en la realidad. No obstante, al disimular su propia operación, al hacerse olvidar en la medida en que logra expresar, el lenguaje hace que el lector ofrezca toda su experiencia de los otros y de los acontecimientos, todas las interrogaciones (Merleau-Ponty, 1971: 33-36). Es decir, el lector no analiza signos, ni estructuras, sino que coproduce el sentido a partir de su propia experiencia, en relación con las expresiones en el texto, relacionadas con las sensaciones y los afectos.

En Placeres se verifican las acciones de los vivos y también las de Cándido ya muerto. Como se verá en el inciso siguiente, los espacios en los que se presenta a Cándido vivo son diferentes de éste. Placeres es el lugar en el que se escuchan las "voces de los muertos" que cohabitan con los vivos, principalmente la del tío. Cándido desea hacerse escuchar entre los vivos, como se dijo antes, y su aparición en Placeres responde a este interés. En este pueblo viven sus descendientes: Isidro y Jeremías, con quienes desea contactar para hacerse presente. También aquí viven Oliva, la esposa de Isidro y madre de Jeremías, y tres personajes más: Marta Licona, la enfermera, que asiste a la madre en el parto de Jeremías, y Omar Vitelo y Colombino Varandas, personajes que no se sabe de dónde llegaron para ayudar a Cándido a volver al mundo de los vivos. Esta inserción del mundo de Cándido muerto en Placeres convierte a este último en un lugar que va perdiendo su vitalidad. Pero la complejidad no se reduce a esta intersección entre los mundos; no es la inserción de Cándido lo que provoca que Placeres sea un sitio desahuciado. Isidro es un hombre que no tiene vida estando vivo y Cándido desea darle esta vitalidad de la que carece. El resultado es, no obstante, la imposibilidad de unos y otros, muertos y vivos, de vivir.

A esta expresión estética de la vida como parte de la muerte y viceversa se le contrapone un esquema de involución, que muestra cómo Placeres transita de una situación aparentemente tolerable, a una en la que se hace imposible habitar el lugar. En algún momento, Isidro dice que en Placeres "volverá a reinar el llano, la soledad, como en un principio" (49), lo cual implica que antes de que ellos arribaran al lugar éste estaba marcado por la desolación. Volver al inicio significa estar en una etapa posterior, pero también, al decir de Isidro, se indica que se volverá al momento anterior; es decir, que hay un movimiento cíclico. No obstante, al leer la novela se percibe la involución, con una distribución lineal; dicho de otra manera, se transita de una situación más o menos vivible a una insoportable. Del nacimiento de Jeremías a la muerte de Isidro, el pueblo tiene ciertas características; trece años después conserva su situación de "tierra tan raída", pero ahora de manera exacerbada. Es Marta, la enfermera, que regresa después de trece años de ausencia, quien experimenta el cambio. Al llegar al pueblo, el narrador dice:

Pese a que Marta conoce todo eso, lo encuentra a su retorno como si jamás lo hubiera visto; le parece una tierra tan raída; un sueño feroz. El cielo, en sus bor- 
des es blanco, vaporoso. No sopla nada de aire. Y lo único que Marta respira es soledad y más soledad. Y la soledad le perfora los huesos. Y desfallece. [...] Marta se endereza en el asiento. Encima del espejismo, la niebla solar es densa. Marta se pregunta, moviendo apenas los labios resecos, si Placeres habrá cambiado, si será ya otro, con el tiempo (75).

La respuesta es sí, el pueblo ha cambiado, se ha modificado y ha transitado de una situación a otra: "pero eso fue en el tiempo que aún no se le secaba la urbe de sus cielos a Placeres, cuando Vitelo, pintor esmerado, no rebasaba los treinta años" (87). Es decir, en el primero momento de la diégesis, cuando se narra el nacimiento de Jeremías y Vitelo tenía esta edad, Placeres no era el sitio en el que se convirtió con los años.

La transformación se verifica también físicamente: "El rebote de los rayos del sol en la tierra blanca, que la deslumbra, no le impide reconocer las fachadas de algunas tiendas. A todas se les ha caído la pintura casi por completo. Están desvencijadas, como cayéndose de hambre. Medio muertas, parece, sin embargo, que una secreta piedad se empeñara en mantenerlas" (78).

La modificación del espacio está dada por el paso del tiempo por las cosas de la ciudad, las casas, sobre todo. Éstas perdieron su color, al punto de no poderse distinguir unas de otras: "Entonces, todas [las casas] eran como vagas figuras de perdidos en la oscuridad del día y del viento, sin querer andar, quietos temerosos" (87). Esta metáfora revela que no son sólo las casas las que han quedado indiferenciadas, sino también que los habitantes han perdido su forma, por lo tanto, su identidad. Destaca aquí la relación entre la desfiguración del espacio y la pérdida de identidad de los personajes.

Veintisiete años más tarde, cuando Jeremías vuelve después de haberse ido todos estos años, el pueblo está en total descomposición: "Son las tres de la tarde, pero el cielo de Placeres está tan turbio que parece nocturno" (117). También en este momento de la narración, el paso del tiempo se describe a partir de la modificación de las casas: "La bodega ha perdido la puerta de atrás y sólo hay un hueco, negro, redondo, en su lugar. [...] Pero su casa está peor, piensa Paniagua. La halló como si hubiera estado en el centro de una de aquellas tormentas de tierra. De una larga tormenta. Del zaguán, quedaban sólo las jambas; de la casa, un cascarón, y del baño, en el fondo del patio, nada" (124).

Jeremías volvió al pueblo porque se lo pidió Cándido, quien continúa haciendo intentos para hacerse escuchar en el mundo de los vivos. No obstante, el tiempo ha destruido el lugar: no queda casi nada.

Los espacios implican un pasado y un futuro. En esta novela, la intención es revocar esta posibilidad. No se sabe por qué los personajes llegaron a Placeres; ellos mismos no lo saben, ni parece importarles. Es simplemente un hecho. "Él, Isidro Paniagua, seguía siendo hombre de casa. Reposado en las costumbres y en el mirar al mundo. Casi no conocía Placeres, ni a su gente" (30). Placeres es un sitio sin historia porque para los personajes no tiene pasado ni futuro. Están ahí como si hubieran sido arrojados de pronto. Además, como ya se dijo, los elementos descritos en el pueblo son tan generales: placita, casas, ca- 
lles, árboles, que pueden formar parte de cualquier pueblo. En este espacio no hay pasado ni futuro. Cuando la enfermera Marta regresa recuerda la condición material de las casas y el clima. También recuerda el nacimiento de Jeremías y las sesiones espiritistas, sin embargo, estos recuerdos no permiten al lector comprender quién es esta enfermera; no se sabe de dónde vino y por qué fue a vivir a Placeres.

Lo mismo ocurre con los amigos de Isidro: "Hasta la fecha Paniagua no sabía cuál era la ocupación de Vitelo, de qué modo gastaba las horas, y tampoco sabía nada de los otros dos. Parecían todos fuereños. Con la casa, la comida, y los intereses, en otro lado. Caídos del cielo" (31). Los amigos tampoco tienen historia y no se sabe cuál es su ocupación. No se cuenta con elementos para poder identificarlos. Ellos llegaron al pueblo para realizar las sesiones espiritistas. También al hablar del hijo es clara la alusión a esta manera de aparecer, de pronto, situado en un espacio ajeno:

Le tocó el cuerpecito, el pelo húmedo de sudor, la frente. Seguía considerándolo un ser extraviado en Placeres. Nada podía arrancarle eso del corazón y sentía —dura y mordiente - pena por Jeremías. [...] Si él no hubiera sido también otro perdido, seco ya, habría podido ayudarle al hijo a encontrar el camino, alguna tierra en el mundo. Qué iba a pasar con el alma de Jeremías el día que él descubriera la falta de alimento para sus raíces; debajo del suelo de Placeres, la piedra impenetrable (47).

La metáfora utilizada para describir a Placeres es precisa: piedra impenetrable. $\mathrm{Si}$ partimos de la idea anterior de que el espacio es espacio vivido, entonces la metáfora es reveladora y confirma el sentido general de esta novela: la existencia misma es impenetrable. Los habitantes de Placeres están perdidos para sí mismos: no saben quiénes son porque no tienen memoria. El lugar sólo afirma esta situación: nada hay en él que permita construir la historia de una vida. En este "desierto", Cándido Paniagua busca un "tornavoz" para hacerse escuchar, no obstante, nada puede resonar ahí, porque no hay tiempo, no hay memoria, no hay historia.

Placeres es el desierto y está "anexado al espacio de dentro" (Bachelard, 1992: 243). Es decir, el espacio es la vastedad en la que se ubica el pueblo, y ésta es, finalmente, la experiencia del sujeto atemorizado con la idea de su finitud. El pueblo es una inmensidad, como lo es el desierto y como lo es la existencia inefable en su ambigüedad.

\section{Cándido Paniagua no es de Placeres}

El personaje principal de la novela, Cándido Paniagua, no nació en Placeres y su aparición por este pueblo ocurrió estando muerto. El narrador dice a propósito de los Paniagua y de Marta Licona: "Los Paniagua no le eran desconocidos a Marta. Hubo un Isidro, de ojos expresivos. Llegado de fuera a Placeres, nunca se asimiló a la vida de Placeres". Y más adelante agrega: "Hubo un Cándido, por los mismos años, pero que no vivía como todo mundo, como su pariente Isidro, sobre la tierra, entre el polvo 
[...]. La ubicación que le confería Vitelo a Cándido Paniagua era en el cielo..." Hacia el final de este fragmento se lee: "Y hubo otro Paniagua: Felipe" (82-83). En Placeres, el espacio en el que Isidro se mueve es la tierra y el espacio de Cándido es el cielo. La ubicación de Felipe no se advierte aquí, aunque se sabe, por otros fragmentos en los que éste es mencionado, que vivió un tiempo en el pueblo donde nacieron los Paniagua y posteriormente se mudó a otro sitio.

En la primera parte de la novela, destacada en el propio texto con el número I, como se dijo anteriormente, el tío Cándido, ya muerto, parece introducirse en el tiempo de Isidro para narrarle algunos sucesos de su infancia y etapa adulta, relacionada, esta última, con los maltratos que recibió de su hermano y cuñada. El tiempo de Cándido en el recuerdo de Isidro es, desde luego, el del tío vivo, pero el espacio es impreciso. No se sabe exactamente en dónde ocurrieron estos hechos, no hay ubicación geográfica ni nombres de los poblados. Se conserva, sin embargo, la idea de clima extremo, propio del desierto.

Aun cuando no se nombran los sitios, es posible diferenciar, por lo menos, dos espacios: uno corresponde al pueblo de donde son originarios los Paniagua y otro se relaciona con el lugar en donde Cándido vivió con su hermano y su cuñada. El primer espacio es configurado con la descripción de tres componentes: la casa de los Paniagua, la placita y la iglesia de Capuchinas. El nombre de este pueblo no se hace explícito; el único lugar que tiene nombre es la iglesia: Capuchinas. Como sabemos, esta denominación remite a una orden religiosa extendida por todo el país. Desde mi perspectiva, en la novela, el nombre no agrega información importante desde el punto de vista histórico, teológico o social. Lo que destaca es la experiencia de la iglesia como espacio, como construcción arquitectónica, con todos sus componentes exteriores e interiores. Este espacio se convierte, así, en elemento principal en la configuración de la identidad de Cándido. No se trata de un sitio al cual se asiste para realizar ciertas actividades, sino del lugar en el que se realiza la existencia de este personaje. La descripción de la experiencia de Cándido con este espacio permite al lector comprender no el dogma, cosa que no interesa en esta novela, sino la soledad.

En la iglesia el personaje encontrará las condiciones para pensarse y sentirse a sí mismo, es decir, para lograr la comprensión sobre su identidad. No se trata de la configuración de una identidad basada en sucesos, sino mostrada a partir de las vivencias. Para expresar esto, se acude a organizaciones simbólicas y metafóricas. En esta novela los personajes no reflexionan sobre la finitud; la estrategia consiste en mostrar la soledad, utilizando formas metafóricas o simbólicas. La iglesia, como obra arquitectónica, con sus contenidos, estatuas de santos, ángeles, con su atmósfera peculiar, se constituye así como expresión simbólica de una vivencia próxima a la soledad existencial.

La importancia de la iglesia se expresa de manera contundente cuando el personaje la relaciona con la casa, metafóricamente: "La iglesia de que hablo era, fue, por años y años, mi verdadera casa" (41). Con esta metáfora se derrumba la idea de la casa paterna, la casa de infancia, para dar lugar a la posibilidad de configurar un sentido de casa que no está ya vinculado con los sucesos precisos, con las anécdotas, sino con la 


\section{$112 \square$ IDENTIDAD, TIEMPO Y ESPACIO EN EL TORNAVOZ DE JESÚS GARDEA}

revelación. Más adelante se lee: "Una tarde en Capuchinas bastará, sobrino. Ésa será la almendra. La suma. [...] Capuchinas se había convertido en mi verdadera casa. Pero también mi verdadera familia se encuentra alli’” (43-44). La casa es para Cándido ese rincón del mundo del que hablaba Bachelard: "la casa es nuestro rincón del mundo. [...] Es un cosmos en toda la acepción del término" (34). Éste es el lugar que hace al personaje y éste se hace en el espacio de su casa. La iglesia es el espacio realmente habitado por Cándido, de ahí que lo considere su casa. En palabras de Bachelard: "todo espacio realmente habitado lleva como esencia la noción de casa" (35).

La "casa" de Cándido, no obstante, no está construida con la memoria y el recuerdo, por lo que el tiempo se convierte en referencia difusa, no forma parte importante de la configuración de la identidad del personaje. Ni la casa ni los habitantes, lo santos y ángeles, se constituyen a partir del pasado y del futuro; no tienen historia, simplemente están ahí sin tiempo, con la eternidad que parece cautivar al personaje. La iglesia es, así, símbolo de ese no transcurso. Para el personaje, el espacio de la iglesia es el espacio constitutivo del silencio y la soledad, que lo conduce a la revelación de esa parte de su existencia que había permanecido oculta para él mismo.

Esta atmósfera que da alegría a Cándido es, paradójicamente, umbría; se relaciona con el silencio, las sombras, la pena: "Huele a incienso y a pena sobre pena; y a oro sombrío y la carne silenciosa de las tallas. Pocas cosas me proporcionaban entonces tanta alegría como estas presencias en el aire quieto de los sábados, sobrino. Pocas. De manera que cuando entro a Capuchinas soy otro: un Paniagua del cielo" (43).

La iglesia no simboliza regocijo o esperanza, sino soledad; el aislamiento en el que vive este personaje y los seres humanos en general. Por medio de la soledad y el arrobo, Cándido se desprende de la temporalidad y experimenta ser etéreo y, de otro modo, eterno.

La iglesia le da esta posibilidad porque es el lugar en el que todo permanece: "Nada ha cambiado en la iglesia. Sombras y silencios son como una sola lluvia circulante entre las columnas, contenida por los muros, por el techo de bóveda y la cúpula" (41-42).

El espacio de esta casa no está constituido sólo por el interior. El exterior es fundamental, porque la iglesia es también su placita, las figuras de la fachada y las palomas. Estos tres elementos forman parte del paisaje que configura la casa de Cándido.

Esta expresión del espacio conduce a la idea de que "la casa es, más aún que el paisaje, un estado de alma", como escribe Bachelard (1992: 104).

El segundo espacio corresponde a la casa en que Cándido vivió con su hermano, Felipe, y con su cuñada. Como ya se dijo arriba, no se precisa la ubicación de este lugar, sólo se menciona el cuarto en el que vive el tío, tras haber sido corrido de la casa por la cuñada, y un patio que comunica la cocina con este pequeño cuarto, aparentemente de servicio. No hay descripciones dimensionales de este espacio ni construcción en secuencias; la presentación es fragmentaria.

En este lugar, el tío muere de frío porque su hermano y su cuñada le quitan el combustible y también aquí resucita y es buscado por sus amigos, los ángeles, los santos. Este espacio es, aparentemente, el lugar de tránsito de la vida a la muerte y a la re- 
surrección. La descripción precaria resalta el suceso: en ese cuarto Cándido muere y también ahí se ha forjado una esperanza con la idea de la resurrección, pero sólo para dudar otra vez. La resurrección no es lo que Cándido esperaba: "Cándido, no hemos traído camino de campo, de jardines; camino suave. Todavía estamos sin desbastar. Todavía es a la soledad lo que nuestros ojos enamoran. Este polvo de la ropa, polvo es, Cándido, del desierto" (59). Para expresar esta situación se utiliza la distinción entre el páramo desértico y el campo fértil, mediante metáforas. Y más adelante se lee: “'Cándido', le dijeron, 'resiste, aférrate a la esperanza. Las flores las trajimos de la placita de Capuchinas. El sacristán las cortó para nosotros. Pero son para ti, Cándido. Hemos atravesado el desierto con ellas" (61).

Los espacios en los que Cándido adquiere consistencia como personaje tienen como correlato externo el desierto. El páramo yermo es la expresión de la extensión de la iglesia: ambos espacios están marcados por la soledad.

\section{Consideraciones finales}

En El tornavoz, el espacio funciona como anclaje del tiempo y permite comprender quiénes son los personajes, más allá de conceptos abstractos e incluso del encadenamiento causal de acciones. La identidad de los personajes no se configura a partir de la construcción de estereotipos ni como individualidades radicadas en su psicología, pero tampoco se trata de la configuración de identidades que cambian con el tiempo, que tienen diversas formas de comprenderse a sí mismas a lo largo de la vida, bajo la égida de su manera de permanecer a pesar de los cambios. Al ser escasas, resulta complicado interpretar las acciones como sucesión encadenada causalmente, que permita comprender las motivaciones de los actos y su sentido. Las acciones ocurren sobre todo en el interior de los personajes; están vinculadas con los sentimientos y los pensamientos, por lo que los acontecimientos escasean y prevalece la idea de extensión espacial del ser. Es decir, estos personajes no tienen un carácter puramente psicologista, debido a que están situados en un espacio específico, un espacio que se codetermina con ellos. Es así como el espacio se convierte en configuración relevante para comprender de qué personajes se habla.

Esta codeterminación entre el espacio y el personaje implica que éstos interiorizan condiciones materiales que son recibidas por ellos de determinada manera y con determinados significados. Es decir, el espacio no es sólo reflejo de una psicología, sino que funciona en relación con los personajes y les permite construir una idea de sí mismos, ya no como historia narrada, sino como situación vivencial.

Así, el espacio no funciona como soporte semántico o reflejo de la psicología, sino como coproductor de la ontología de estos personajes. El clima es el indicador más importante en este sentido. El calor y el frío intensos son amenazas concretas, que están ahí, y que dejan a los habitantes de Placeres en la indefensión. En el desierto se vive como muerto. 


\section{$114 \square$ IDENTIDAD, TIEMPO Y ESPACIO EN EL TORNAVOZ DE JESÚS GARDEA}

Placeres es un sitio sin pasado y sin futuro. El espacio no tiene aquí sus tiempos, pasado o futuro, porque los personajes han perdido la memoria. Arrancados de sus espacios originarios y de la posibilidad de recordar el transcurso de sus vidas, se conciben a sí mismos como seres "perdidos" en ese mundo de Placeres, que es el mundo del desierto y que también es la extensión del adentro.

En relación con los espacios en los que habita Cándido Paniagua sucede algo semejante. En el primer caso, cuando el personaje decide que su casa es la iglesia, renuncia a sus orígenes familiares, para vincularse con este nuevo hogar, que remite a lo incorruptible. Cándido busca permanecer, y encuentra un espacio arquitectónico y simbólico en el cual hacerlo. La iglesia le proporciona la soledad y el silencio que necesita y también le brinda el encuentro con la paz añorada, con el reposo que le ofrecen los santos y ángeles que han permanecido por siglos en sus sitios.

El otro espacio en el que habita Cándido es la casa del hermano. Aquí, en la dimensión terrenal, él experimentará tristeza y dolor. En este espacio el personaje muere y resucita para continuar su búsqueda ahora en Placeres, entre los vivos.

Placeres, así como la iglesia, parecen ser otras expresiones del tornavoz, de ese espacio que busca el personaje para resonar entre los vivos y hacerlos vivir.

\section{Obras citadas}

AINSA, Fernando. 2005. Espacio literario y fronteras de la identidad. Costa Rica: Editorial de la Universidad de Costa Rica.

BaChelard, Gaston. 1992. Poética del espacio. México: FCE.

DREW, Alejandrina. 1985. Ficción y realidad en El tornavoz de Jesús Gardea. Trabajo de grado de maestría no publicado. Texas: The University of Texas at El Paso.

FouCAult, Michel. 1992. Microfísica del poder. Madrid: Ediciones de la Piqueta.

GardeA, Jesús. 2004. El tornavoz. México: Ediciones Sin Nombre.

GARRIDO DOMíNGUEZ, Antonio. 1996. El texto narrativo. Madrid: Síntesis.

HuTCHEON, Linda. 1992. "Ironía, sátira, parodia. Una aproximación pragmática a la ironía". De la ironía a lo grotesco (en algunos textos hispanoamericanos). México: UAM-Iztapalapa.

Merleau-Ponty, Maurice. 1971. La prosa del mundo. Madrid: Taurus.

RicArdou, J. 1971. Pour une théorie du nouveau roman. París: Éditions du Seuil.

Ricoeur, Paul. 1986. Del texto a la acción. Ensayos de hermenéutica II. México: FCE.

VILLAMIL, Elvira. 1997. "Espacio indeterminado en el discurso lacónico de El tornavoz de Jesús Gardea”. Revista de Literatura Mexicana Contemporánea, vol. II, núm. 4. Pp. 58-69. 